Research Article

\title{
On Total Vertex Irregularity Strength of Hexagonal Cluster Graphs
}

\author{
Nurdin Hinding $\mathbb{D}^{1},{ }^{1}$ Hye Kyung Kim, ${ }^{2}$ Nurtiti Sunusi $\mathbb{D}^{3},{ }^{3}$ and Riskawati Mise ${ }^{4}$ \\ ${ }^{1}$ Department of Mathematics, Faculty of Mathematics and Natural Sciences, University of Hasanuddin, \\ Makassar 40133, Indonesia \\ ${ }^{2}$ Department of Mathematics Education, Daegu Catholic University, Gyeongsan 38430, Republic of Korea \\ ${ }^{3}$ Department of Statistic, Faculty of Mathematics and Natural Sciences, University of Hasanuddin, Makassar 40133, Indonesia \\ ${ }^{4}$ Department of Mathematics, Maros Muhammadiyah University, Maros, Indonesia
}

Correspondence should be addressed to Nurdin Hinding; nurdin1701@gmail.com

Received 4 August 2020; Revised 29 December 2020; Accepted 4 January 2021; Published 23 January 2021

Academic Editor: Sergejs Solovjovs

Copyright (c) 2021 Nurdin Hinding et al. This is an open access article distributed under the Creative Commons Attribution License, which permits unrestricted use, distribution, and reproduction in any medium, provided the original work is properly cited.

For a simple graph $G$ with a vertex set $V(G)$ and an edge set $E(G)$, a labeling $f: V(G) \bigcup E(G) \longrightarrow\{1,2, \cdots, k\}$ is called a vertex irregular total $k$-labeling of $G$ if for any two different vertices $x$ and $y$ in $V(G)$ we have $w t(x) \neq w t(y)$ where $w t(x)=$ $f(x)+\sum_{u \in V(G)} f(x u)$. The smallest positive integer $k$ such that $G$ has a vertex irregular total $k$ - labeling is called the total vertex irregularity strength of $G$, denoted by tvs $(G)$. The lower bound of tvs $(G)$ for any graph $G$ have been found by Baca et. al. In this paper, we determined the exact value of the total vertex irregularity strength of the hexagonal cluster graph on $n$ cluster for $n \geq 2$. Moreover, we show that the total vertex irregularity strength of the hexagonal cluster graph on $n$ cluster is $\left(3 n^{2}+1\right) / 2$.

\section{Introduction}

A graph labeling is an assignment of integers from 1 to $n$, for the vertices, edges, or both. Graph labelings have been used in many applications like communication network addressing, software testing, information security, technology and sports tournament scheduling, and coding theory problems including the design of good radar location codes, missile guidance codes, and convolution codes.

We consider the finite undirected graph $G$ without loops and multiple edges with vertex set $V(G)$ and edge set $E(G)$. The degree of a vertex $x$ is the number of edges that have $x$ as an endpoint, and the set of neighbors of $x$ is denoted by $N(x)$. If the domain of the labeling function $f(x)$ is the vertex set or the edge set, the labeling is called, respectively, vertex labeling or edge labeling. If the domain is $V(G) \bigcup E(G)$, then we call the labeling a total labeling.

A labeling $f: E(G) \longrightarrow\{1,2,3, \cdots, k\}$ is called an edge $k$-labeling of $G$. The associated vertex weight of a vertex $x \in V(G)$ under an edge $k$-labeling $f$ is defined as $w t(x)=\sum_{v \in N(x)} f(x v)$. Chartrand et al. [1] introduced an edge $k$ - labeling of a graph $G$ such that $w t(x) \neq w t(y)$ for all vertices $x, y \in V(G)$ with $x \neq y$. Such labelings were called irregular assignments, and the irregularity strength $s(G)$ of a graph $G$ is known as the minimum $k$ for which $G$ has an irregular assignment using labels at most $k$. The irregularity strength $s(G)$ can be interpreted as the smallest integer $k$ for which $G$ can be turned into a multigraph $G$, by replacing each edge by a set of at most $k$ parallel edges, such that the degrees of the vertices in $G^{\prime}$ are all different.

In this paper, we consider for a total $k$ - labeling of $G$, that is, $f: V(G) \bigcup E(G) \longrightarrow\{1,2,3, \cdots, k\}$. The associated vertex weight of a vertex $x \in V(G)$ under a total $k$ - labeling $f$ is defined as $w t(x)=f(x)+\sum_{v \in N(x)} f(x v)$. A total $k$ - labeling $f$ is defined to be a vertex irregular total $k$-labeling of $G$ if for every two different vertices $x$ and $y$ of $G, w t(x) \neq w t(y)$. The minimum positive integer $k$ for which $G$ has a vertex irregular total $k$ - labeling is called the total vertex irregularity strength of $G$, denoted by tvs $(G)$. In Figure 1, there are a two total labeling of $P_{4}$, one of is a vertex irregular total 3-labeling of $P_{4}$ and the 


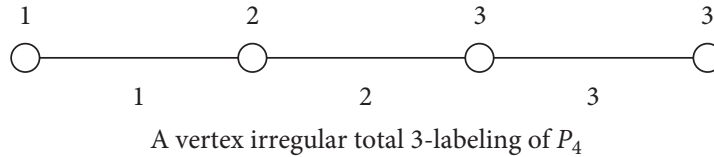

(a)

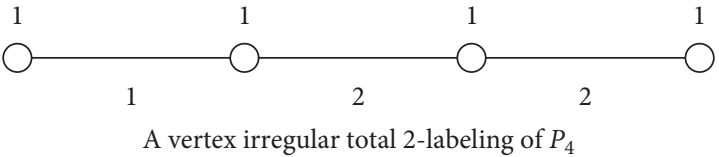

(b)

Figure 1: A vertex irregular total $k$-labeling of $P_{4}$ for $k=2$ and $k=3$.

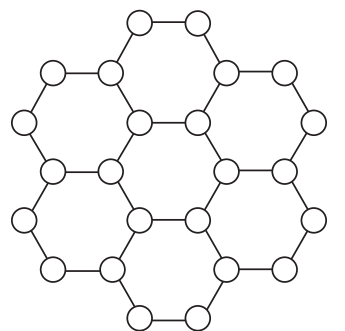

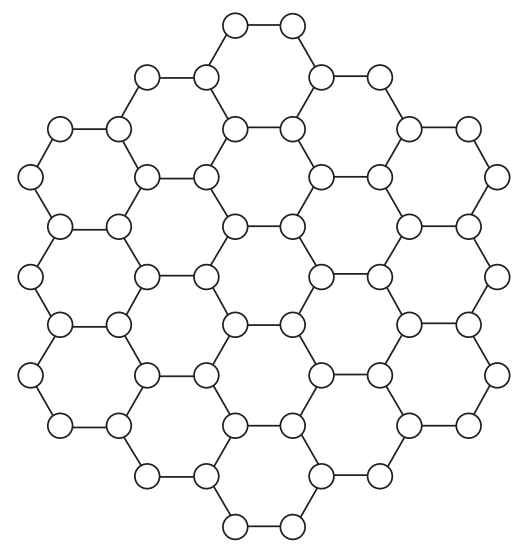

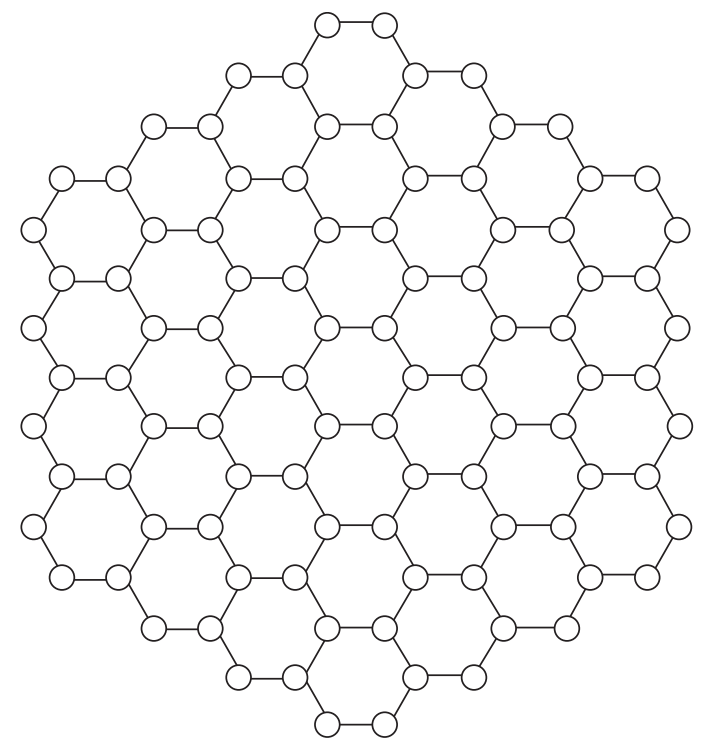

Figure 2: Hexagonal cluster graphs HC(2), HC(3), and HC(4).
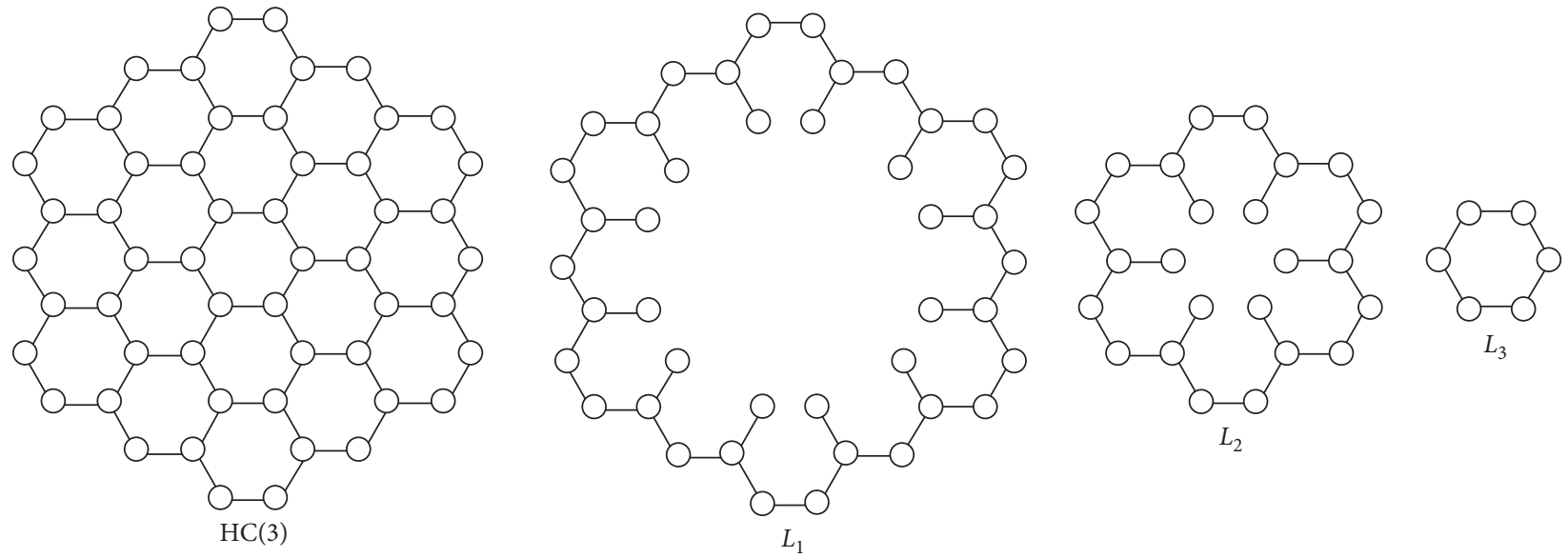

Figure 3: Hexagonal cluster graphs $\mathrm{HC}(3)$ with their layers $L_{1}, L_{2}$, and $L_{3}$.

Step 1. For $i=1+3(k-1)$ where $k=1,2, \cdots, 2 n$, label $e^{\prime}, e^{\prime \prime}$, and $x_{i}$ with $(i+2) / 3$ where $e^{\prime}$ and $e^{\prime \prime}$ are edges incident to vertex $x_{i}$ Step 2. For $i=1+3(k-1)$ where $k=1,2, \cdots, 2 n$, label edges between $x_{i}$ and $x_{i+1}$ by $f\left(x_{i}\right)+1$ except for the labeling edges in Step 1 Step 3. Label all edges between $x_{1}$ and $x_{6 n-2}$ by $f\left(x_{6 n-2}\right)+1=2 n+1$

Step 4 . Label the remaining edges of $L_{1}$ by $4 n-3$ 


$$
\begin{aligned}
\mathrm{K}_{2 \mathrm{t}-1}=\left(\mathrm{S}_{2 \mathrm{t}-1}+2-\mathrm{K}_{2 \mathrm{t}-2}\right) / 3 \text {, Step } A \text {. Label all of edges of the outer cycle in } L_{t} \text { for } 2 \leq t \leq n-1 \text { by where } K_{2}=4 n-3 \\
\text { Step } B \text {. Label all of the remaining edges from } L_{t} \text { for } 2 \leq t \leq n-2 \text { by } K_{2 t}=\left(S_{2 t}+2-2 K_{2 t-1}\right) / 2 \\
\text { Step } C \text {. Label all of the remaining edges in } \mathrm{HC}(n) \text { by }\left(3 n^{2}+1\right) / 2
\end{aligned}
$$

Algorithm 2: Algorithm for labeling all other edges in $\mathrm{HC}(\mathrm{n})$.

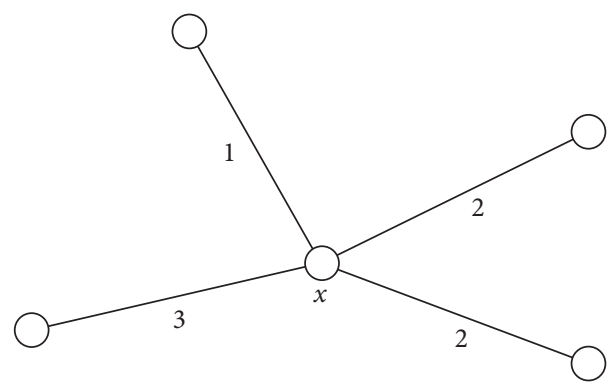

FIgURE 4: The temporary weight of $x$ is 8 .

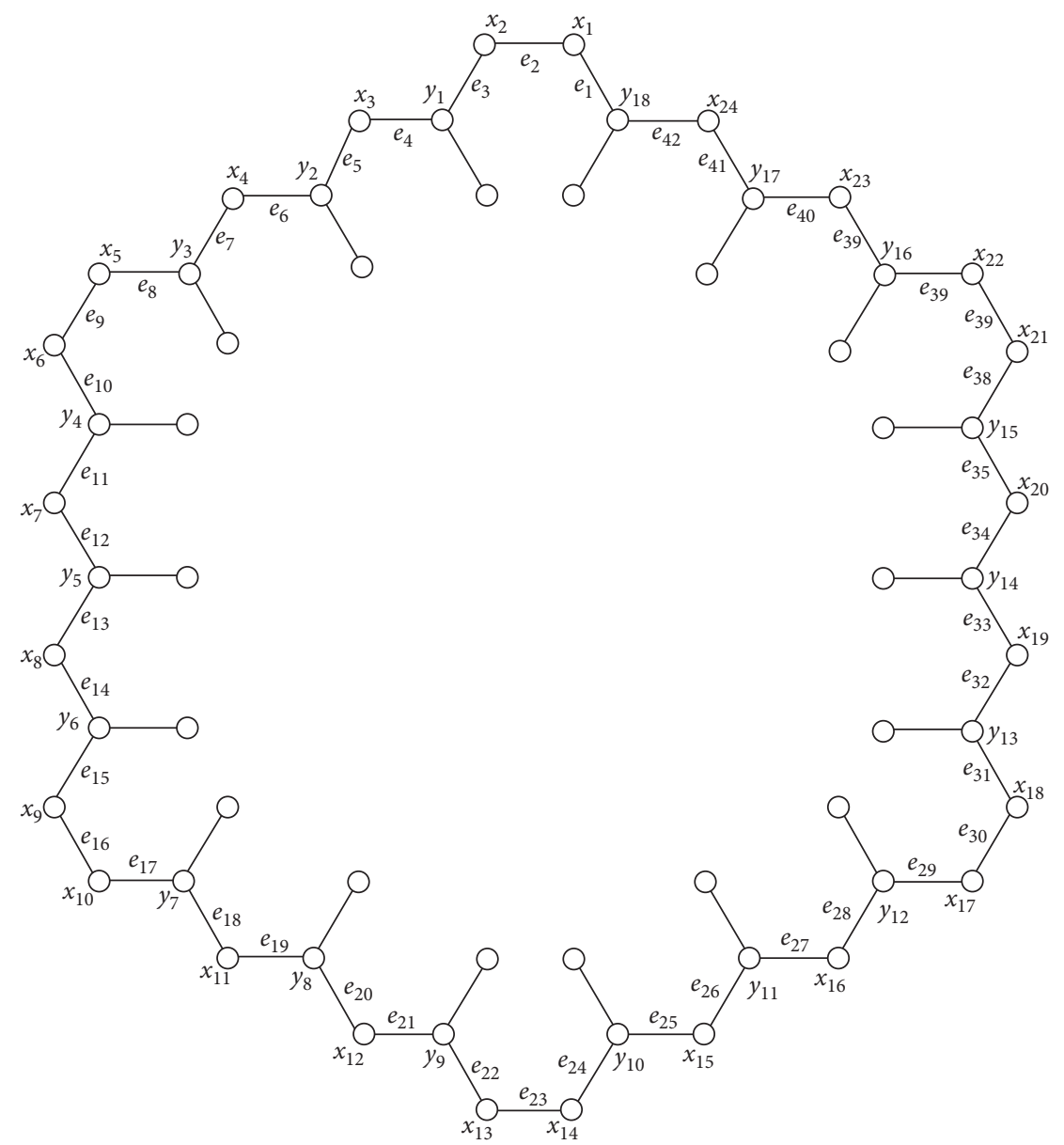

Figure 5: The name of elements of the outer cycle $C_{42}$ of $L_{1}$ in $\mathrm{HC}(4)$.

other is a vertex irregular total 2-labeling of $P_{4}$. However, $P_{4}$ does not have a vertex irregular total vertex 1-labeling. Such that the minimum positive integer $k$ for which $P_{4}$ has a vertex irregular total $k$ - labeling is 2 , or the total vertex irregularity strength of $P_{4}$ is 2 .
Baca et al. [2] in 2007 started to investigate the total vertex irregularity strength of a graph, an invariant analogous to the irregularity strength for total labelings. There are not many graphs for which the exact values of their total vertex irregularity strength are known. Baca et al. [2] have determined the total 
Step 1. For $i=1+3(k-1)$ where $k=1,2,3,4,5,6,7,8$, label $e^{\prime}, e^{\prime \prime}$, and $x_{i}$ with $(i+2) / 3$ where $e^{\prime}$ and $e^{\prime \prime}$ are edges incident to vertex $x_{i}$ (see Figure 6)

Step 2. For $i=1+3(k-1)$ where $k=1,2,3,4,5,6,7,8$, label edges between $x_{i}$ and $x_{i+1}$ by $f\left(x_{i}\right)+1$ except for the labeling edges in Step 1

Step 3. Label all edges between $x_{1}$ and $x_{22}$ by $f\left(x_{24}\right)+1=9$ except for the labeling edges in Step 1 (see Figure 7)

Step 4. Label the remaining edges of $L_{1}$ by 13 (see Figure 8 )

Algorithm 3: Algorithm for labeling all edges of the outer cycle of L1 of HC(4).

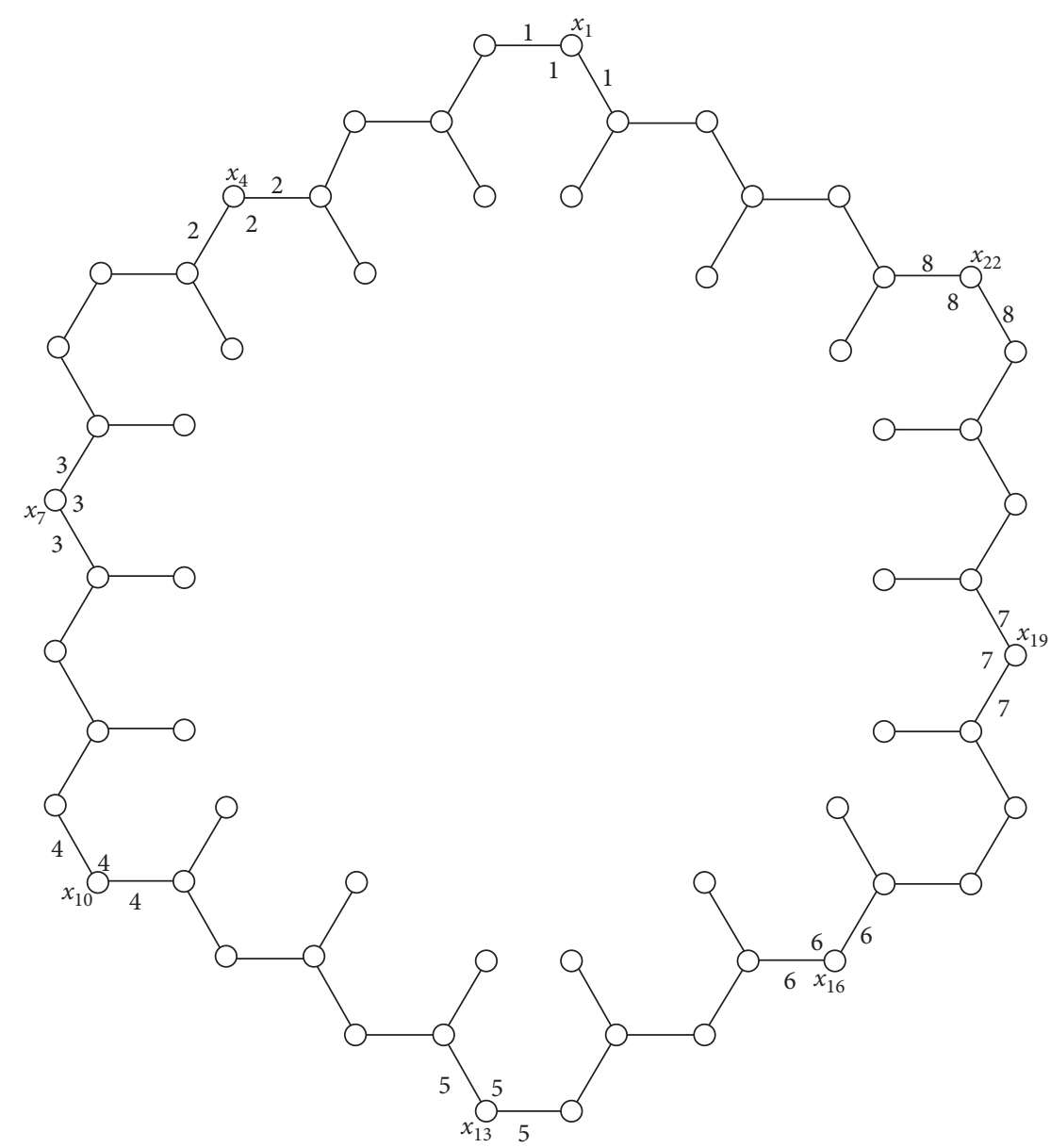

Figure 6: The labels of some vertices and edges of $L_{1}$ in $\mathrm{HC}(4)$.

vertex irregularity strengths for some classes of graphs, namely, cycles, stars, and prisms. Nurdin et al. have determined the total vertex irregularity strengths of a disjoint union of $t$ copies of a path [3], tree graphs [4], and caterpillar graph [5]. Nurdin and Kim have determined the total vertex irregularity strength of splitting graphs of stars [6].

In this paper, we determine exact value of the total vertex irregularity strength of the hexagonal cluster graph with $n$ cluster for $n \neq 2$.

\section{Hexagonal Cluster Graph}

In this section, we give the definition of hexagonal cluster graphs. The hexagonal cluster graph with $n \geq 2$ cluster, denoted by $\mathrm{HC}(n)$, where $\mathrm{HC}(1)$ isomorphs to $C_{6}$, is obtained by adding as many as $6(n-1)$ cycle $C_{6}$ on the outer path of $\mathrm{HC}(n-1)$. Figure 2 demonstrates hexagonal cluster graphs $\mathrm{HC}(2), \mathrm{HC}(3)$, and $\mathrm{HC}(4)$.

Some interconnection networks are designed, and some are borrowed from nature. For example, hypercubes, complete binary trees, butterflies, and torus networks are some of the designed architectures. Grids, hexagonal networks, honeycomb networks, and diamond networks, for instance, bear resemblance to atomic or molecular lattice structures. They are called natural architectures. The advancement of large scale integrated circuit technology has enabled the construction of complex interconnection networks. Besides that, the hexagonal cluster graphs have been studied as models of organic compounds build up entirely from benzene rings, social networks, and wireless sensor 


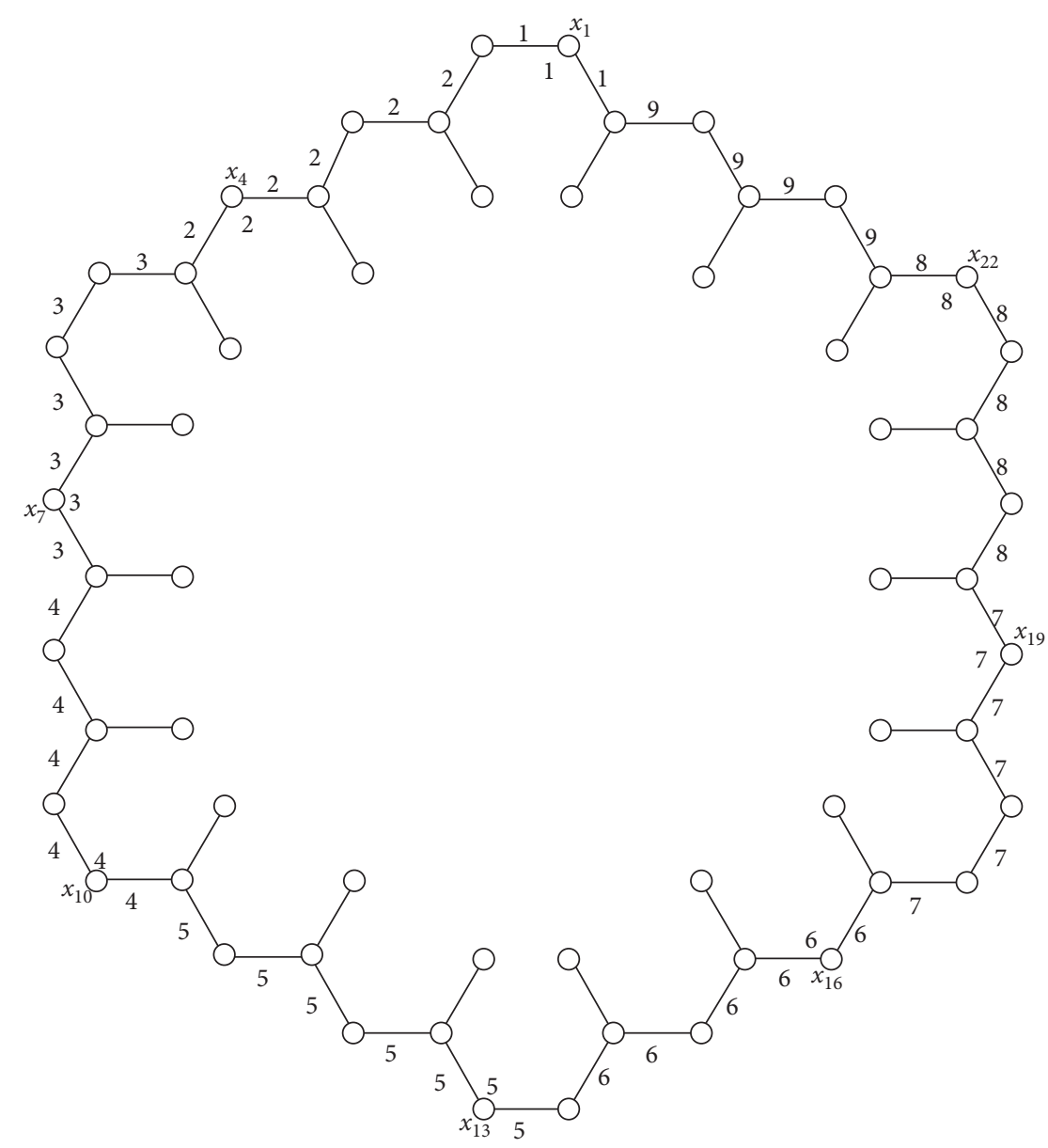

Figure 7: The labels of some vertices and edges of $L_{1}$ in $\mathrm{HC}(4)$.

networks. Graph theory provides a fundamental tool for designing and analyzing such networks [7-11].

In [2], Baca et al. studied the lower bound of $\operatorname{tvs}(G)$ for any graph $G$ as follows.

Theorem 1. If $p$ is the number of vertices of any graph $G, \delta$ is the minimum degree of vertex, and $\Delta$ is the maximum degree of vertex of $G$, then

$$
\operatorname{tvs}(G) \geq \frac{p+\delta}{\Delta+1}
$$

\section{Results and Discussion}

In this paper, we have proved that the total vertex irregularity strength of the hexagonal cluster graph (network) with $n$ cluster is equal to its lower bound in Theorem 1 .

Theorem 2. For $n \geq 2$, we have

$$
\operatorname{tvs}(\mathrm{HC}(n))=\frac{3 n^{2}+1}{2} \text {. }
$$

Proof. Since the number of vertices of $\mathrm{HC}(n)$ is $6 n^{2}$, the minimum degree of vertex is 2 and the maximum degree of vertex is 3; by using (1) in Theorem 1, we found that

$$
\operatorname{tvs}(\mathrm{HC}(n)) \geq \frac{3 n^{2}+1}{2} \text {. }
$$

To find that $\operatorname{tvs}(\mathrm{HC}(n)) \leq\left(3 n^{2}+1\right) / 2$, we have to construct a vertex irregular total $k$-labeling on $\mathrm{HC}(n)$ where $k=\left(3 n^{2}+1\right) / 2$ as follows.

Note that there are $n$ layers in $\mathrm{HC}(n)$. Layer $i$ is denoted by $L_{i}$. For illustration, in Figure 3 , there are 3 layers in $\mathrm{HC}(3)$.

In $L_{1}$, there is an outer cycle $C_{12 n-6}$ of $\mathrm{HC}(n)$ which consists of $6 n$ vertices of degree 2 denoted by $x_{i}(i=1,2, \cdots, 6 n), 6(n-1)$ vertices of degree 3 denoted by $y_{j}(j=1,2, \cdots, 6(n-1))$, and $12 n-6$ edges denoted by $e_{t}(t=1,2, \cdots, 12 n-6)$.

Now, consider all vertices and all edges in the outer cycle $C_{12 n-6}$ of $\mathrm{HC}(n)$ sequentially.

To label some of vertices and all edges in $L_{1}$, we use Algorithm 1 as follows.

To label all of edges in $L_{t}$ for $2 \leq t \leq n-1$ and for $3 \leq i \leq 2 n-3$ define 


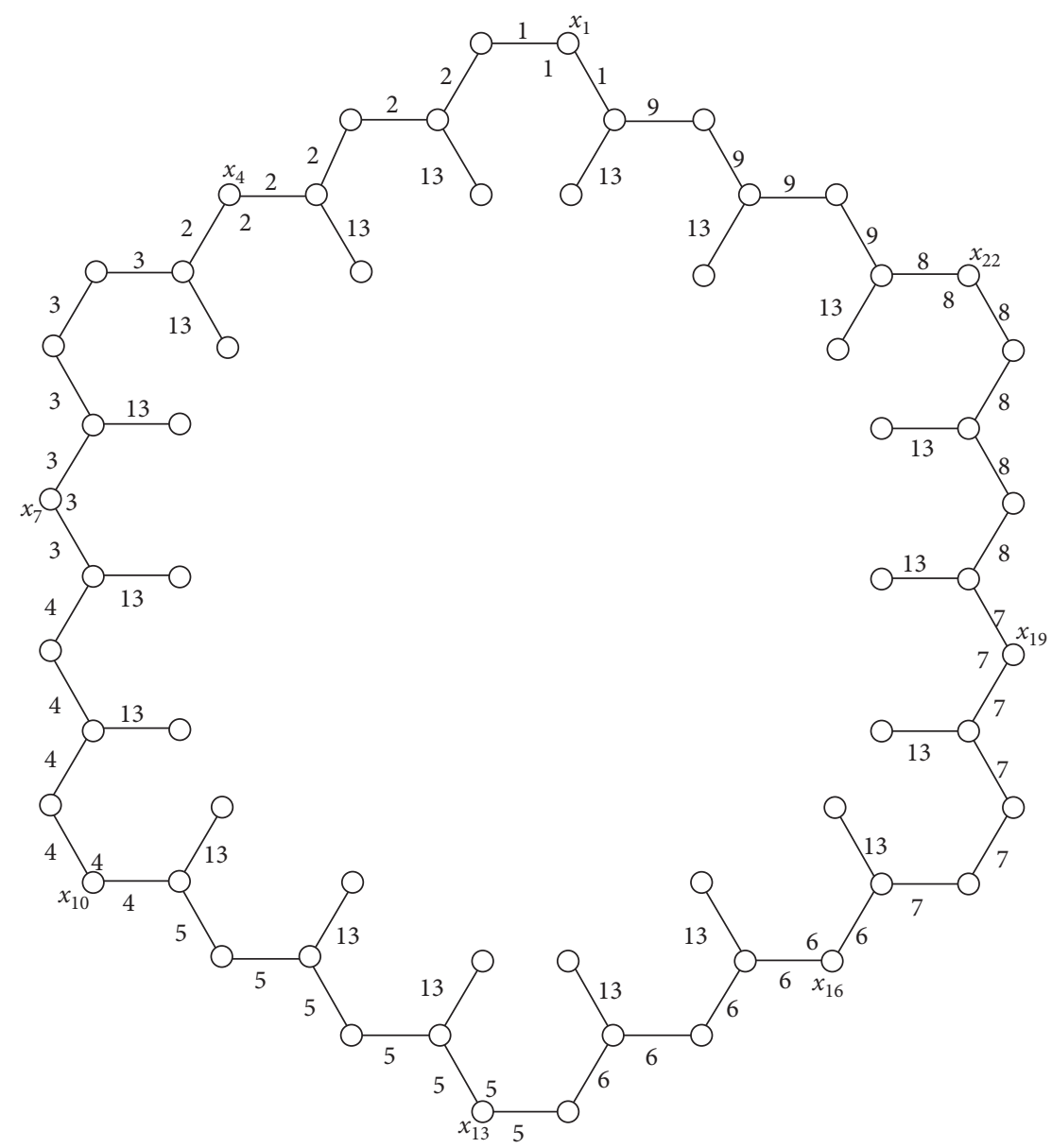

FIgURE 8: The labels of some vertices and edges of $L_{1}$ in $\mathrm{HC}(4)$.

Step A. Label all edges of the outer cycle in $\mathrm{L}_{2}$ by using Algorithm 2, i.e., $K_{3}=\left(S_{3}+2-K_{2}\right) / 3$ where $K_{2}=13$,i.e., $K_{3}=(60+2-$ $13) / 3=17$ (see Figure 9) and all edges of the outer cycle in $L_{3}$ by $K_{5}=\left(S_{5}+2-K_{4}\right) / 3=(84+2-20) / 3=22$ (see Figure 10) Step B. Label all of the remaining edges from $L_{2}$ by using Algorithm 2, i.e., $K_{4}=\left(S_{4}+2-2 K_{3}\right) / 2=(72+2-2.17) / 2=20($ see Figure 11)

Step C. Label all of the remaining edges in $\mathrm{HC}(4)$ by $\left(3.4^{2}+1\right) / 2=25$ (see Figure 12)

Algorithm 4: Algorithm for labeling all other edges in HC(4).

$$
\begin{gathered}
S_{i}=6 n i-\frac{3}{2}\left(i^{2}-1\right), \quad \text { for } i \text { is odd, } \\
S_{i}=6 n i-\frac{3}{2} i^{2}, \quad \text { for } i \text { is even, }
\end{gathered}
$$

and use Algorithm 2 as follows.

All of edges of $\mathrm{HC}(n)$ are already labeled, but vertices have not labeled yet. Next, label all of vertices using the following method. The temporary weight of a vertex $x$ is the number of label of all edges incident to $x$, denoted by $w(x)$. For example, the temporary weight of a vertex $x$ in Figure 4 is 8 .

Order the temporary weight of all vertices and rename them by $z_{1}, z_{2}, \cdots, z_{6 n^{2}}$ such that $2=w\left(x_{2}\right)=w\left(z_{1}\right) \leq w$ $\left(z_{2}\right) \leq \cdots \leq w\left(z_{6 n^{2}}\right)$. For $i=2,3, \cdots, 6 n$, define

$$
\begin{aligned}
f\left(z_{i}\right) & =\max \left\{1, w t\left(z_{i-1}\right)+1-w\left(z_{i}\right)\right\}, \\
w t\left(z_{i}\right) & =w\left(z_{i}\right)+f\left(z_{i}\right),
\end{aligned}
$$

where $w t\left(z_{1}\right)=3$ is the total weight of vertex $z_{1}$.

Based on equation (6), we have that

the weights of all vertices in $\mathrm{E}(\mathrm{HC}(n))$ are distinct.

Besides that, from Algorithms 1 and 2, we can see that

$$
\text { the largest label on } \mathrm{HC}(n) i s \frac{3 n^{2}+1}{2} \text {. }
$$

Based on Statements (7) and (8), we conclude that the function construction with Algorithms 1 and 2 is a total 


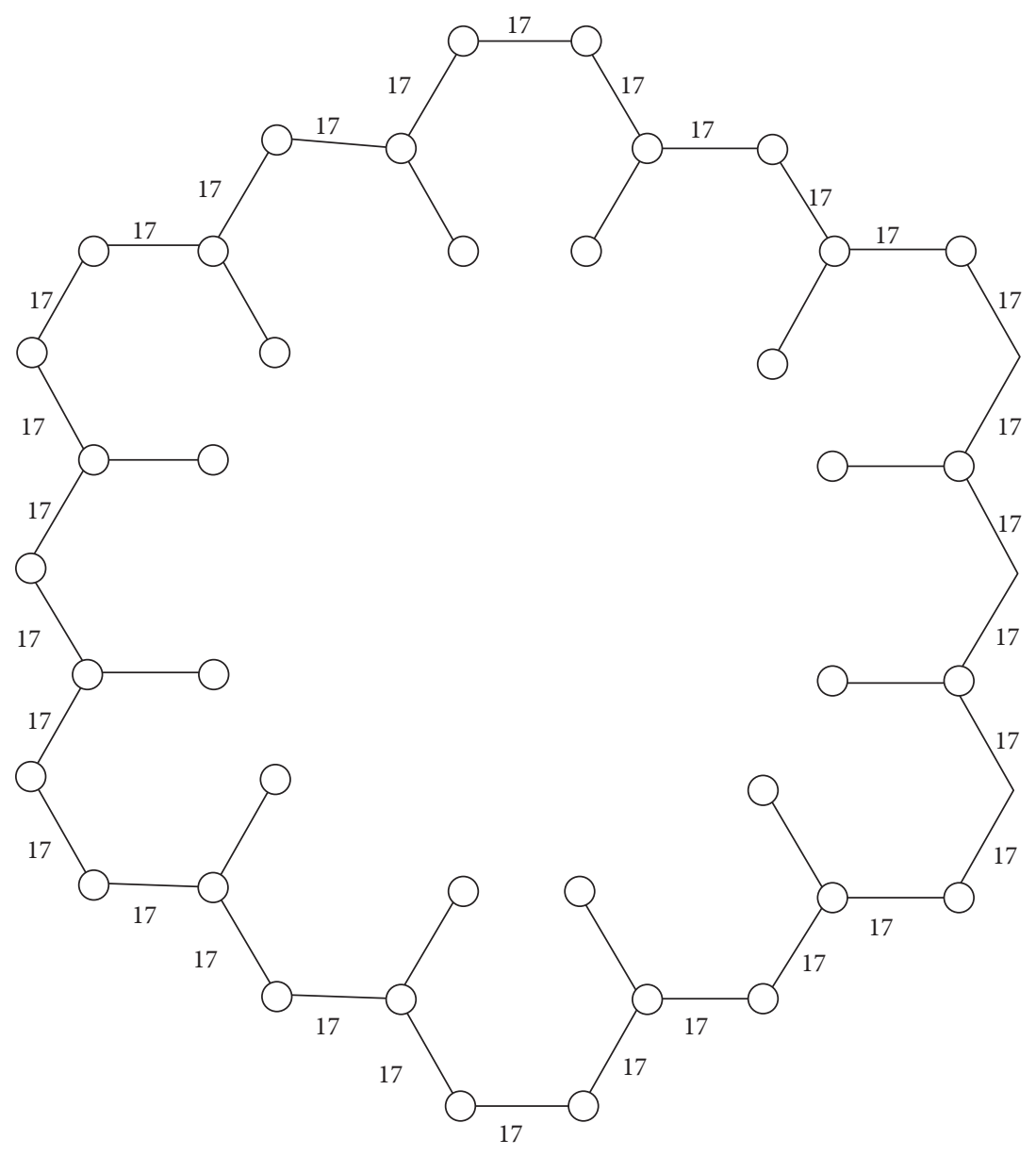

Figure 9: The labels of all edges of the outer cycle $C_{30}$ of $L_{2}$ in $\mathrm{HC}(4)$.

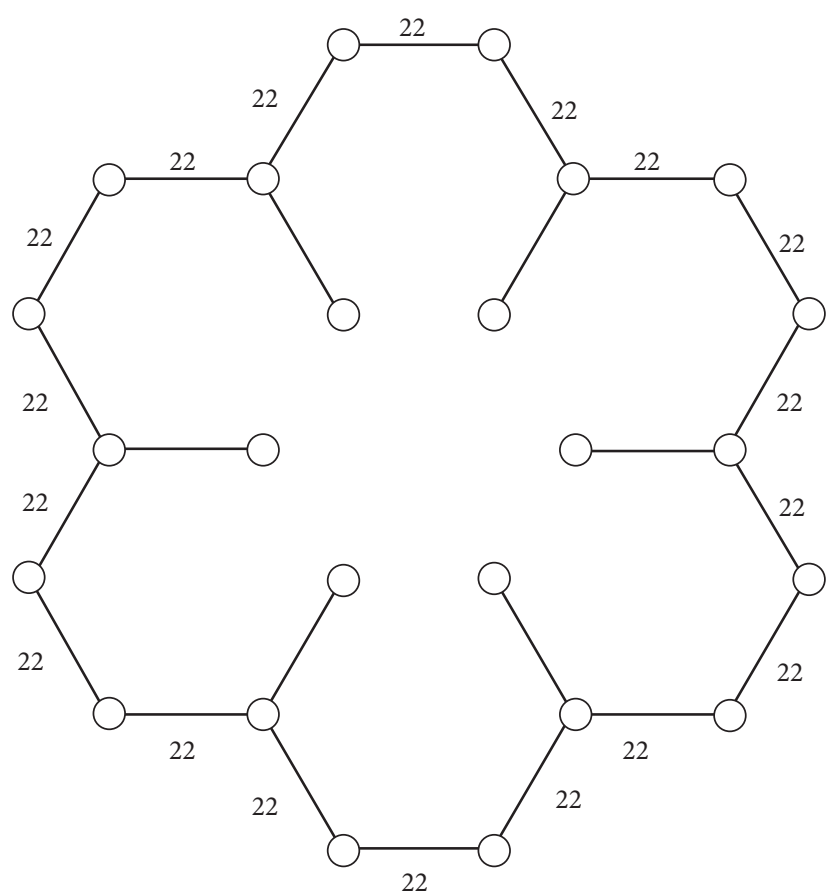

Figure 10: The labels of all edges of the outer cycle $C_{18}$ of $L_{3}$ in $\mathrm{HC}(4)$. 


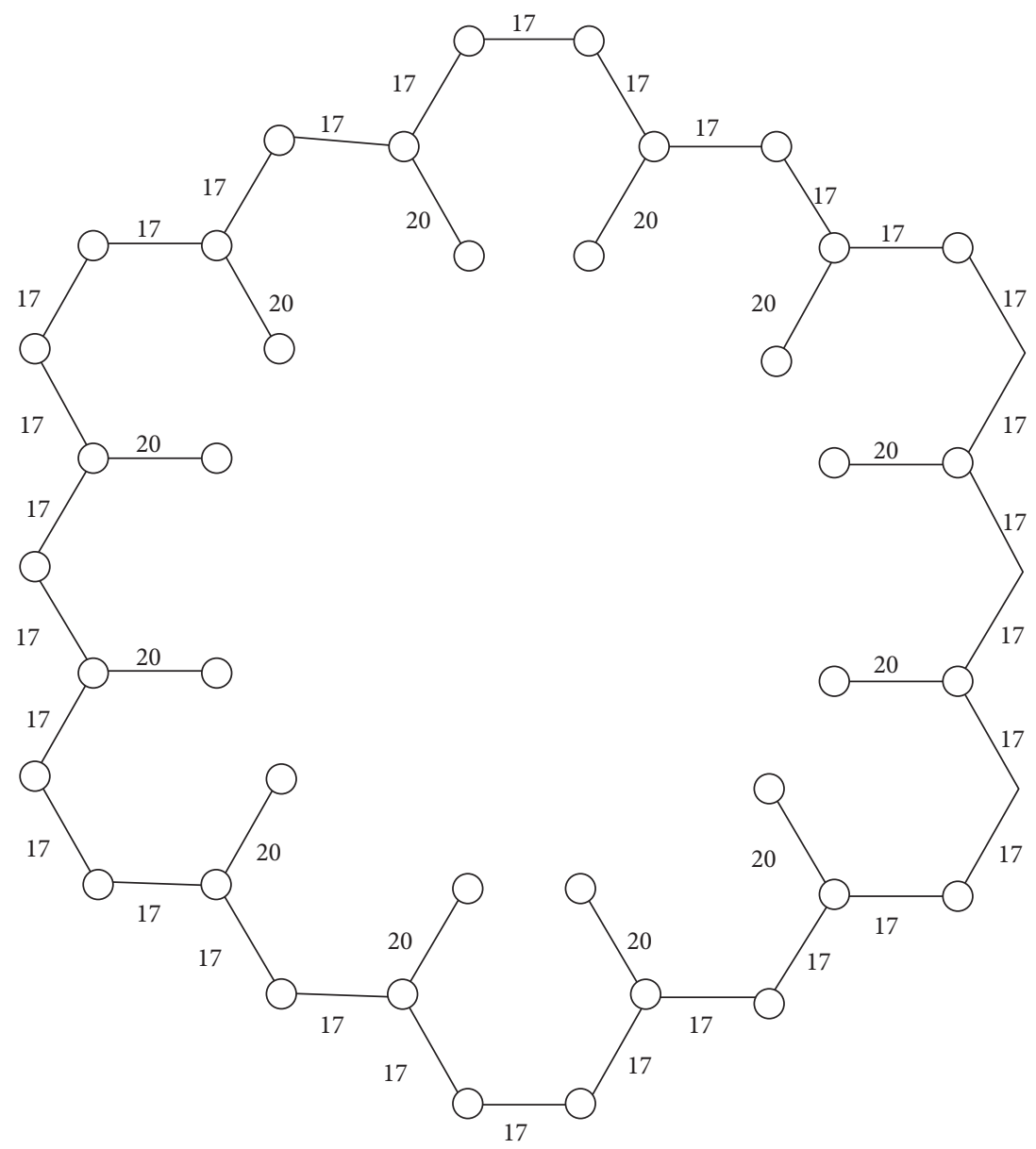

FIgURE 11: Layer 2 with edge labels.

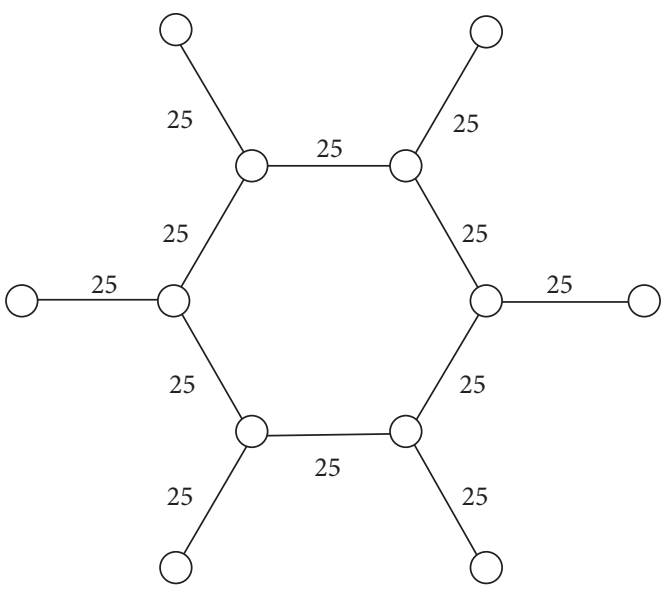

FIGURE 12: Layer 4 with edge labels.

vertex irregular $k$ - labeling on $\mathrm{HC}(n)$ where $k=\left(3 n^{2}+\right.$ $1) / 2$. This shown as follows:

$$
\operatorname{tvs}(\mathrm{HC}(n)) \leq \frac{3 n^{2}+1}{2} \text {. }
$$

Based on equations (3) and (9), we find equation (2), i.e., $\operatorname{tvs}(\mathrm{HC}(n))=\left(3 n^{2}+1\right) / 2$.
As illustration, we shall use Algorithms 1 and 2 to construct a total vertex irregular 25-labeling on $H C$ (4). The first step, all the vertices and all the edges on the outer cyrcle $\mathrm{C}_{42}$ of $\mathrm{HC}(4)$ are named sequentially as in Figure 5.

Now, we use Algorithm 3 to label some of vertices and all edges in $L_{1}$ as follows. 
For $3 \leq \mathrm{I} \leq 5$, using equations (4) and (5), we have $S_{3}=$ $24.3-(3 / 2)\left(3^{2}-1\right)=60, \quad S_{4}=24.4-(3 / 2) 4^{2}=72$, and $S_{5}=24.5-(3 / 2)\left(5^{2}-1\right)=84$. follows.

Then, use Algorithm 4 to label all edges in $L_{2}$ and $L_{3}$, as

\section{Conclusions}

In this paper, we obtained the precise values for the total vertex irregularity strength of the hexagonal cluster graphs $\mathrm{HC}(n)$, for all $\mathrm{n} \geq 2$. Furthermore, we show that the hexagonal cluster graphs $\mathrm{HC}(n)$ is an example that the lower bound in lcite\{BJMR07\} is sharp. In the future, we are interested in computing the exact value for the total vertex (or edge) irregularity strength of grids, hexagonal networks, and honeycombs networks.

\section{Data Availability}

The data used to support the findings of this study are included within the article.

\section{Conflicts of Interest}

The author(s) declare that they have no conflicts of interest regarding the publication of this paper.

\section{Acknowledgments}

This research was supported by the Basic Science Research Program, National Research Foundation of Korea, Ministry of Education, (NRF-2018R1D1A1B07049584) and Basic Research Superior College, Directorate of Research and Community Service, Ministry of Research, Technology and Higher Education, Republic of Indonesia (007/SP2H/AMD/ LT/DRPM/2020).

\section{References}

[1] G. Chartrand, M. S. Jacobson, J. Lehel et al., "Irregular networks," Congressus Numerantium, vol. 81, pp. 113-119, 1988.

[2] M. Baca, S. Jendrol, M. Miller, and J. Ryan, "On irregular total labellings," Discrete Mathematics, vol. 307, pp. 1378-1388, 2007.

[3] Nurdin, A. N. M. Salman, N. N. Gaos, and E. T. Baskoro, "On the total vertex-irregular strength of a disjoint union of tcopies of a path," Journal of Combinatorial Mathematics and Combinatorial Computing, vol. 71, pp. 227-233, 2009.

[4] E. T. B. Nurdin, A. N. M. Salman, and N. N. Gaos, "On the total vertex irregularity strength of trees," Discrete Mathematics, vol. 71, pp. 227-233, 2010.

[5] Nurdin, M. Zakir, and Firman, "Vertex-irregular labeling and vertex-irregular total labeling on caterpillar graph," International Journal of Applied Mathematics and Statistics, vol. 40, no. 10, pp. 99-105, 2013.

[6] Nurdin and H. K. Kim, "Irregular labeling on transportation network of splitting graphs of stars," Proceedings of the Jangjeon Mathematical Society, vol. 22, no. 1, pp. 103-108, 2019.
[7] O. Al-Mushayt and A. Ahmad, "On the total edge irregularity strength of hexagonal grid graphs," Australasian Journal of Combinatorics, vol. 53, pp. 263-271, 2012.

[8] I. Rajasingh and S. T. Arockiamary, "Total edge irregularity strength of honeycomb torus networks," Global Journal of Pure and Applied Mathematics, vol. 13, no. 4, pp. 1135-1142, 2017.

[9] J. Sedlacek, "Problem 27 in thoery of graphs and its applications," Proceedings of the Symposium Smolenice, pp. 163167, 1963.

[10] I. Stojmenovic, "Honeycomb networks: topological properties and communication algorithms," IEEE Transactions on Parallel and Distributed Systems, vol. 8, no. 10, pp. 1036-1042, 1997.

[11] I. Tarawneh, R. Hasni, and A. Ahmad, "On the edge irregularity strength of grid graphs," AKCE International Journal of Graphs and Combinatorics, vol. 17, no. 1, pp. 414-418, 2020. 